\title{
Cuando las cenicientas se unen: docencia cooperativa y gestión de las desigualdades de género en Ciencia Política
}

\author{
Igor Ahedo $^{a}$, Iraide Alvarez \\ a Departamento de Ciencia Política y de la Administración (Universidad del País Vasco / Euskal Herriko \\ Unibertsitatea)igor.ahedo@ehu.eus \\ b Departamento de Ciencia Política y de la Administración (Universidad del País Vasco / Euskal Herriko \\ Unibertsitatea) iraide.alvarez@ehu.eus
}

\section{Resumen}

La incorporación de la perspectiva de género en educación necesita de la visibilización de las desigualdades en el aula a fin de superar la ceguera de género. La ciencia política muestra que solo el tránsito de la consideración privada de las desigualdades a la consideración de su carácter público y estructural permite procesos de politización que acaban generando normas de obligado cumplimiento. En este texto, presentamos una experiencia en la que la perspectiva de género se incorpora al curriculum formal en la asignatura de Fundamentos del Análisis Político del Grado de Ciencia Política y Gestión Pública de la UPV/EHU. A partir del cuento de La Cenicienta, se explicitan las normas de género que provocan una desigual distribución del trabajo "productivo y público" de una parte, y "reproductivo y privado" de otra. Tras contextualizar la desigualdad, se aprovecha la presentación de las metodologías docentes para evidenciar que la desigualdad está presente en el aula. Finalmente, gracias a la visibilización y consideración pública del problema, se invita al alumnado a diagnosticar y gestionar estas desigualdades en el marco de la metodología del Aprendizaje basado en proyectos que vertebra la asignatura.

\section{Palabras clave:}

Aprendizaje basado en proyectos - género - enseñanza cooperativa - igualdad - inclusión - Ciencia Política - Universidad - Alumnado

\section{Introducción: de la ley a la práctica docente}

Los marcos legislativos europeos, estatales y autonómicos son claros al mostrar una actitud proactiva en la definición normativa de la necesidad de incorporar la perspectiva de género en educación superior. La Ley Orgánica 4/2007 por la que se modifica la LOU 6/2001 otorga a este espacio educativo un papel central en la transmisión de valores. Señala expresamente que la búsqueda de "una sociedad tolerante e igualitaria, en la que se respeten los derechos y libertades fundamentales y de igualdad entre hombres y mujeres, debe alcanzar, sin duda, a la Universidad". El RD 1393/2007, de 29 de octubre por el que se establece la ordenación de las enseñanzas universitarias oficiales, señala que "la formación en cualquier actividad profesional debe contribuir al conocimiento y desarrollo de los Derechos Humanos, los principios democráticos, los principios de igualdad entre mujeres y hombres, de solidaridad, de protección medioambiental, de accesibilidad universal y diseño para todos, y de fomento de la cultura de la paz”. En lo que respecta al marco nnormativo de nuestra comunidad de referencia, la Comunidad Autónoma del País Vasco, la Ley (4/2005) de igualdad entre mujeres y hombres establece expresamente 
que "las universidades velarán por que en la docencia y en los trabajos de investigación sobre las diferentes áreas de conocimiento se integre la perspectiva de género, se haga un uso no sexista del lenguaje y se incorpore el saber de las mujeres y su contribución social e histórica al desarrollo de la Humanidad".

A pesar de todo, investigaciones de académicas están evidenciando la existencia de profundas resistencias que impiden que los preceptos normativos sedimenten en una efectiva y real incorporación de la perspectiva de género superadora de las desigualdades en el espacio universitario (Verge et al., 2018). De forma más explícita, la Evaluación cualitativa del cumplimiento de la Ley de Igualdad (Ahedo et al., 2016), es clara a la hora de evidenciar una falta de actitud proactiva por parte de la estructutra universitaria. Ello hace que los avances queden a expensas de un "claro voluntarismo en el trabajo de las personas participantes en estas estructuras", razón por la que se reclaman recursos, mecanismos de vertebración y estrategias de liderazgo político (Ahedo et al., 2017: 157). El análisis cualitativo sobre el que se basa la evaluación del cumplimiento de esta Ley permite evidencia, en las palabras de las persona participantes, las claves apuntadas. De una parte la lógica voluntarista que guía el avance: "La integración de la perspectiva de género en nuestra universidad pasa porque las mujeres feministas que aquí trabajan investigan en esta cuestión y la incorporan a la docencia" (ibíd., 162). De otra, la ausencia de tratamiento específico de las cuestiones de género en disciplinas claves en la reproducción de los estereotipos desigualitarios: "es inaudito que no haya ninguna asignatura sobre estos temas en carreras como comunicación audiovisual, periodismo, educación (...) que son carreras que crean discurso, que reproducen valores y roles". La directora de igualdad también es clara en esta cuestión al señalar cómo no solo no se ha avanzado, sino que se ha retrocedido. Señala (...) "cómo la transversalidad de estos temas no solo no garantiza que se aborden, sino que es la excusa para que no se trabajen. (...) La valoración, en consecuencia, es muy crítica. Se apunta que hasta este momento no ha podido haber cambios en los grados hasta su acreditación, pero que ahora que se podría hacer, tampoco se hace (...) A la hora de encontrar responsabilidades, algunas de las entrevistadas achacan esta cuestión a la falta de voluntad, otras remiten al machismo benevolente: "No creo que haya nadie impidiendo que estas cosas se trabajen, pero en la práctica, no se trabajan. Es como un velo que hace que no se avance. Pero no es algo que esté solo presente en la UPV/EHU'.

El intento de superar esta lógica voluntarista se ha asentado en una serie de acciones contempladas en el III Plan de Igualdad de la Universidad del País Vasco / Euskal Herriko Unibertsitatea (elaborado posteriormente a la evaluación de la Ley). Disciplinas como Ciencia Política y Sociología han incorporado al plan de estudios asignaturas relacionadas con el género (concretamente Teoría Política Feminista en $4^{\circ}$ del grado de Ciencia Política y Gestión Pública); se ha implementado una estrategia formativa dirigida a toda la comunidad, concretada en una oferta de 5 cursos online de formación en género en los que han partidipado más de 2000 personas, así como un curso específico para el profesorado titulado "Avanzando en una docencia con perspectiva de género" impartido quienes suscriben este texto, posteriormente ampliado al profesorado del G9.

Este curso es uno de los resultados de un Proyecto de Innovación Educativa financiado por el Vicerrectorado de Innovación y Responsabilidad Social, pilotado por un equipo de investigadoras y docentes del grupo de investigación en democracia participativa Parte Hartuz. Entre las acciones de este PIE está la realización de un diagnóstico de la incorporación de la perspectiva de género en los grados de la Facultad de Ciencias Sociales y de la Comunicación, así como la implementación de una encuesta para conocer el interés del alumnado en las cuestiones de género. Además de este estudio cuantitativo (Zugaza et al., 2020), una parte del equipo, que en su docencia utiliza estrategias de enseñanza cooperativa, ha diseñado una estrategia de abordaje al género que sitúa al alumnado en el centro del diagnóstico y gestión de las desigualdades. Esta estrategia, cuyos objetivos, desarrollo y resultados preliminaries presentamos a 
continuación, se incardina en dos de las acciones previstas en el III Plan de Igualdad de la UPV/EHU: 1. Fomentar el trabajo conjunto mediante comunidades ampliadas de aprendizaje en torno al quinto Objetivo de Desarrollo Sostenible (ODS): igualdad de género; 2. Alinear el desarrollo de las competencias en materia de género e igualdad con la estrategia de enseñanza-aprendizaje (Ikaskuntza, Ikerkuntza, Iraunkortasuna/Aprendizaje, Investigación, Sostenibilidad).

Ciertamente, el acercamiento a la perspectiva de género y la satisfacción de las recomendaciones identificadas es más practicable en ciertas disciplinas como la de la Ciencia Política. Concretamente, los contenidos de la asignatura de Fundamentos del Análisis Político ${ }^{1}$ encajan como un un guante de terciopelo a la hora de poder abordar la perspectiva de género, en la medida en que uno de los resultados de aprendizaje esperados es precisamente que el alumnado sea "capaz de comprender el carácter público de las desigualdades e identificar sus expresiones visibles, latentes y ocultas".

A la hora de abordar este objetivo, los contenidos de la asignatura incorporar la perspectiva de género en un temario en el que se introducen marcos interpretativos de la teoría política feminista, estudiando cómo las dinámicas ocultas de poder subyacen a todas las relaciones sociales (De Lauretis, 1989; Butler, 1997; Lamas, 2002; Young, 2002; Segato, 2016), incluidas las educativas (Cassese y Bos, 2013; Lois y Alonso, 2014; Verge et al. 2018; Benito y Verge, 2020). Esta perspectiva entiende la desigualdad como la manifestación encarnada (Esteban, 2004) del sistema de dominación sexo-género.

En consecuencia, la propia temática interpela al alumnado y al profesorado. Por ello, en coherencia con la exigencia que se plantea al alumnado (observar las expresiones ocultas del poder), el profesorado debe interrogarse sobre la forma en que las desigualdades se expresan en su ejercicio profesional: en nuestro caso, pasamos nuestra práctica docente por la rúbrica de la teoría que enseña al alumnado. Esta teoría señala que la desigualdad de género se asienta en una socialización diferenciada que relega a las mujeres a papeles emocionales, privados y reproductivos, reservando para los hombres el espacio racional, público y productivo (Carrasco, 1992; Bourdieu, 2000; Martínez-Palacios, 2018a). Si se asume que el género "importa" (García-Pérez, 2011), también en la práctica docente, ésta debe hacer frente a unos retos que afectan al currículum formal. En el caso de la asignatura Fundamentos del Análisis Político esto se concreta, entre otros aspectos, en: 1. Desafiar el modelo masculino como única referencia de conocimiento (Freixas, 2006) visibilizando en los contenidos la experiencia femenina en fenómenos políticos como la correduría y la gestión de conflictos (Ormazabal y Gorostidi, 2017), la crisis de los cuidados (Isaksen, 2016), el peso de lo reproductivo en la acumulación capitalista (Federici, 2010) o las plurales expresiones del ejercicio del poder (Hernando, 2018). 2. Corregir la ausencia del teorizar femenino en los abordajes de la disciplina, reconociendo el papel de las politólogas y sociólogas en los corpus teóricos aludiendo y presentado acercamientos de la teoría política feminista y la sociología del género ${ }^{2}$.

De la misma forma, y no menos importante, es necesario atender a aspectos del currículum oculto, que implica: 1. Poner en cuestión el sistema de evaluación, prestando cuidadosa atención a resultados de investigaciones que abordan el coeficiente simbólico negativo con el que se asocia lo femenino por el cuerpo docente, tanto en las retroalimentaciones al alumnado (Castillo y Corral, 2011) como en la evaluación sesgada de los ejercicios (Moss-Racusin, 2012), e incluso en el reconocimiento de la excelencia (Bengoetxea, 2015). 2. Tomar en consideración los resultados investigaciones que están prestando atención a los patrones de desigualitarios presentes en el aula (Freixas y Fuentes-Guerra, 1997),

\footnotetext{
${ }^{1}$ Impartida conjuntamente al alumnado del grado de Ciencia Política y del Grado de Sociología durante el primer curso en la UPV/EHU.

${ }^{2}$ Una base de datos de la producción bibliográfica en teoría política y género de los últimos cinco años está disponible en https://generopolitica.aecpa.es/recursos/novedades-editoriales-y-publicaciones/
} 
especialmente en lo relativo a la seguridad en el uso de la palabra en los debates (Bolibar-Cruz et al., 2018), reconociendo el origen estructural de la renuencia a participar en grupos grandes por parte de las mujeres (Martínez-Palacios, 2018a). 3. Prestar especial atención a las consecuencias que los roles reproductivos pueden estar generando una sobrecarga de trabajo a las mujeres en las dinámicas grupales que se hace invisible a los ojos del docente (Zugaza et al., 2020) y no se reconocen en valoraciones que prestan atención a aspectos socialmente identificados como productivos, tales como las presentaciones orales, en los que los alumnos presentan más auto-confianza (Bolívar-Cruz et al., 2018).

Este último aspecto que remite a las interacciones del alumnado, se presenta como el más complejo de abordar, ya que la lógica autónoma del trabajo grupal dificulta el control directo y permanente del profesorado. Así, partimos de la hipótesis de que las estrategias grupales, cada vez más populares, pueden ser puntos ciegos para la incorporación de la perspectiva de género en las aulas.

Por ello, aprovechamos la experiencia docente acumulada en la implementación de metodologías cooperativas asentadas en este caso en el Aprendizaje basado en proyectos. Durante el segundo cuatrimestre, el alumnado, organizado en grupos, tiene que realizar un Proyecto en el que se parte del supuesto activador de que forman parte de un grupo de asesores a opositores en procesos de democratización. Como miembros de la organización Movilization, deben realizar 3 informes a la oposición democrática identificando el marco estrucutral del conflicto (Tema 2), las relaciones entre los actores implicados (Tema 3) y el peso de las identidades y las ideologías presentes (Tema 4). Además de estos informes, individualmente deben realizar una serie de ejercicios prácticos. Para desarrollar esta dinámica cooperativa, el alumnado, además de las tareas centradas en los contenidos, tiene que desplegar estrategias orientadas a velar por el buen funcionamiento del proceso. Entre ellas, destaca la elaboración de un convenio de grupo, firmado por todos los componentes, en el que se identifican los roles (a través de encuestas de autodiagnóstico), los compromisos individuales y grupales, los sistemas de resolución de conflictos, así como los elementos sobre los que sostendrán la evaluación a sus compañeros y compañeras. A este convenio se añade un cuaderno de viaje en el que identifican los avances y problemas en relación con el objetivo formativo del Proyecto, pero también respecto del desarrollo del grupo, identificando bloqueos organizativos y/o emocionales.

Estas herramientas, como propondremos, pueden servir para que el alumnado asuma el protagonismo en la identificación y gestión de las desigualdades de género. Para ello, previamente, hay que demostrar que la desigualdad existe. Así, en la presentación de la asignatura, tras identificar los elementos que explicitan las formas de poder oculto, y tras centrar el sentido de la política como el mecanismo que permite la gestión pública de problemas previamente analizados como privados, se aprovecha una simulación de las estrategias cooperativas para evidenciar, en la práctica, que la desigualdad existe en el aula. Esta estrategia muestra al alumnado que los repartos de roles en términos reproductivos para las chicas y productivos para los chicos, no son consecuencia de aptitudes personales, sino de procesos de socialización que requieren de una gestión politica asentada en normas; normas que la metodología del Abp permite incorporar, gestionar y trabajar de la mano de las herramientas de organización presentadas, y de los mecanismos de diagnóstico que identificamos en este trabajo.

\section{Objetivos: superando las normas de género en el trabajo en grupos}

A tal efecto, la experiencia que se presenta se apoya en el Aprendizaje basado en proyectos para:

- Evidenciar que el trabajo en grupos en el aula puede ser un punto ciego a la gestión de las desigualdades con perspectiva de género por parte del profesorado comprometido en la medida en que las normas de género quedan fuera de su control y provocan en la interaccióin del 
alumnado una distribución desigual de la carga de trabajo que orienta a las alumnas a tareas reproductivas y no visibles, mientras que permite a los alumnos mostrar una actitud más proactiva y visible que no hace justicia a su menor carga de trabajo.

- Mostrar cómo las metodologías del Aprendizaje basado en proyectos pueden ser aprovechadas para que el alumnado sea capaz de autodiagnosticar las desigualdades de género e incorporar en la lógica de la interdependencia recírproca la gestión de esta realidad.

Para ello, es necesario abordar una serie de objetivos específicos, tales como:

- Identificar estrategias que incorporen al curriculum formal herramientas interpretativas de las desigualdades, especialmente las normas de género que subyacen a la division de género que focaliza en las mujeres lógicas reproductivas y privadas mientras que aporta a los hombres una centralidad en el espacio identificado como productivo y público.

- Diseñar estrategias metodológicas que permitan la visibilización de este marco teórico desde la práctica personal, vinculada a la experiencia vital del alumnado en las aulas.Todo ello al objeto de mostrar que las desigualdades responden a una lógica pública, pero que no pueden ser gestionadas si se interpretan en clave privada.

Finalmente, y desde un punto de vista aplicado, esta propuesta remite a las recomendaciones del Consejo de Europa Rec (2007) a los Estados miembros sobre las normas y los mecanismos de igualdad entre mujeres y hombres identifica, entre los elementos que indican la voluntad política de los Estados y su compromiso a favor de la igualdad entre mujeres y hombres, que hacemos ahora extensibles a la práctica docente. Concretamente, nos referimos a las recomendaciones iii., vi., vii. y $\mathrm{x}$.

- Incluir explícitamente "el principio de igualdad entre mujeres y hombres (...) y de una perspectiva de género en (...) las políticas educativas";

- Realizar "seguimiento regular de los programas de enseñanza, el contenido de los temas, las normas pedagógicas, los recursos de enseñanza y aprendizaje, así como la organización de las clases (...) con el fin de eliminar los estereotipos de género en todos los niveles del sistema educativo";

- "garantizar el desarrollo, en condiciones de igualdad, de las competencias personales que los estereotipos han tendido a asignar a uno de ambos sexos, tales como la autoestima, el trabajo en equipo, la toma de la palabra en público o la resolución pacífica de los conflictos";

- Proponer un método de "seguimiento y valoración regulares de la participación de (...) mujeres y hombres en (el) sistema educativo".

\section{Desarrollo de la innovación: cuando las cenicientas se unen}

El aprendizaje grupal es una práctica cada vez más extendida y valorada en la universidad (Swanson et al., 2019). Existe un consenso a la hora de considerar estas estrategias (colaborativas, cooperativas, basadas en proyectos/problemas) como pioneras para lograr un aprendizaje significativo y profundo (Doyle, 2008; DeLotell et al., 2010). Ahora bien, ciertos estudios identifican una serie de problemas estructurales derivados de la falta de hábito, coordinación y desarrollo de este modelo (Hillyard, 2010). Aquí nos interesan los que se refieren a la recepción por el alumnado, que identifica riesgos asociados al clima de colaboración (Lizzio y Wilson, 2005): sensación de un reparto injusto de la carga de trabajo (Liden et al., 1985), tensiones derivadas de la gestión de conflictos y los liderazgos (Robinson et al., 2015; Gillespie et al., 2006a), o frustraciones causadas por la baja autoestima del alumnado o su percepción de falta de control sobre la temática de trabajo (Micari y Pazos, 2014; Gillespie et al., 2006b).

(cc) BY-NC-ND 2021, Universitat Politècnica de València

Congreso In-Red (2021) 
Partimos de la hipótesis de que estas desigualdades pueden verse atravesadas por el género. Grupos de discusión con alumnas de $4^{\circ}$ curso de Ciencia Política (que han trabajado el género en la carrera) verbalizan cómo las dinámicas grupales son tierra abonada para la asunción de roles reproductivos por su parte, con la sobrecarga de trabajo tanto práctico como mental que supone (Zugaza et al., 2020). Recurren a términos como "madre" y "secretaria" para definir su papel en estos grupos.

A la hora de comprender este fenómeno, asumimos la perspectiva teórica que se trabaja en los contenidos de clase para explicar al alumnado fenómenos sociológicos como el "techo de cristal" o el "suelo pegajoso". Hablamos de la influencia de las normas de género incorporadas a través de la socialización (Martínez, 2018a), que generan pautas de comportamiento que atraviesan el curriculum informal alimentado la visibilidad productiva y pública masculina y la invisibilidad del trabajo reproductivo femenino no valorado.

- De una parte, las exigencias de discreción y de rigidez explican las dificultades que sienten las alumnas para expresarse en público (Bolívar-Cruz et al., 2018).

- Por su parte, habitus (Bourdieu, 1988) femeninos asentados en normas como la empatía, la disciplina y la entrega al otro explican que gran parte de la carga de los trabajos grupales en el aula recaiga en las mujeres, reforzando forma roles reproductivos y privados.

En la práctica de las aulas, el "techo de cristal" se concreta en la dificultad para hacerse visibles; mientras que la lógica del "suelo pegajoso" puede relegar a las alumnas a un trabajo muchas veces invisible a los ojos del cuerpo docente. Sin embargo, existe un rechazo por parte del alumnado a identificar estas realidades desiguales (Freixas y Fuentes Guerra, 1997), que se achacan a rasgos personales como la timidez o la capacidad organizativa. La mirada es privada. El problema no se reconoce como público. Y la necesidad de reconocimiento público de las desigualdades es precisamente el centro de la Ciencia Política (Vallès, 2006). Se abre una vía en el curriculum formal para atacar el punto ciego a la perspectiva de género detectado en el trabajo en grupo.

En la asignatura de Fundamentos del Análisis Político, de acuerdo con los manuales (Caminal, 2006; Vallès, 2006), se define la política como el mecanismo para la gestión pública de desigualdades estructurales. En la jornada de presentación se utiliza el cuento de la Cenicienta como metáfora para ejemplificar el tránsito de la consideración privada a la interpretación pública de las desigualdades como motor de una política que concreta soluciones colectivas en normas de obligado cumplimiento.

Se reflexiona sobre lo que subyace a ideas centrales como la "orfandad", el "fregar suelos", el "acceso al príncipe" o "la magia". Se acuerda que el origen de la subordinación de la Cenicienta no es la mala suerta, sino unas relaciones de poder estructurales. Se apunta que la toma de conciencia del carácter público de las desigualdades emerge cuando las Cenicientas ven que no están sola (Cohen y Arato, 2006). Así, pueden unirse a través de procesos de politización que concretan demandas de cambio político. Se concluye que "cuando las cenicientas se unen se cambia el cuento y el príncipe entendido como poder se define como res pública, cosa pública" (Ahedo, 2006). 


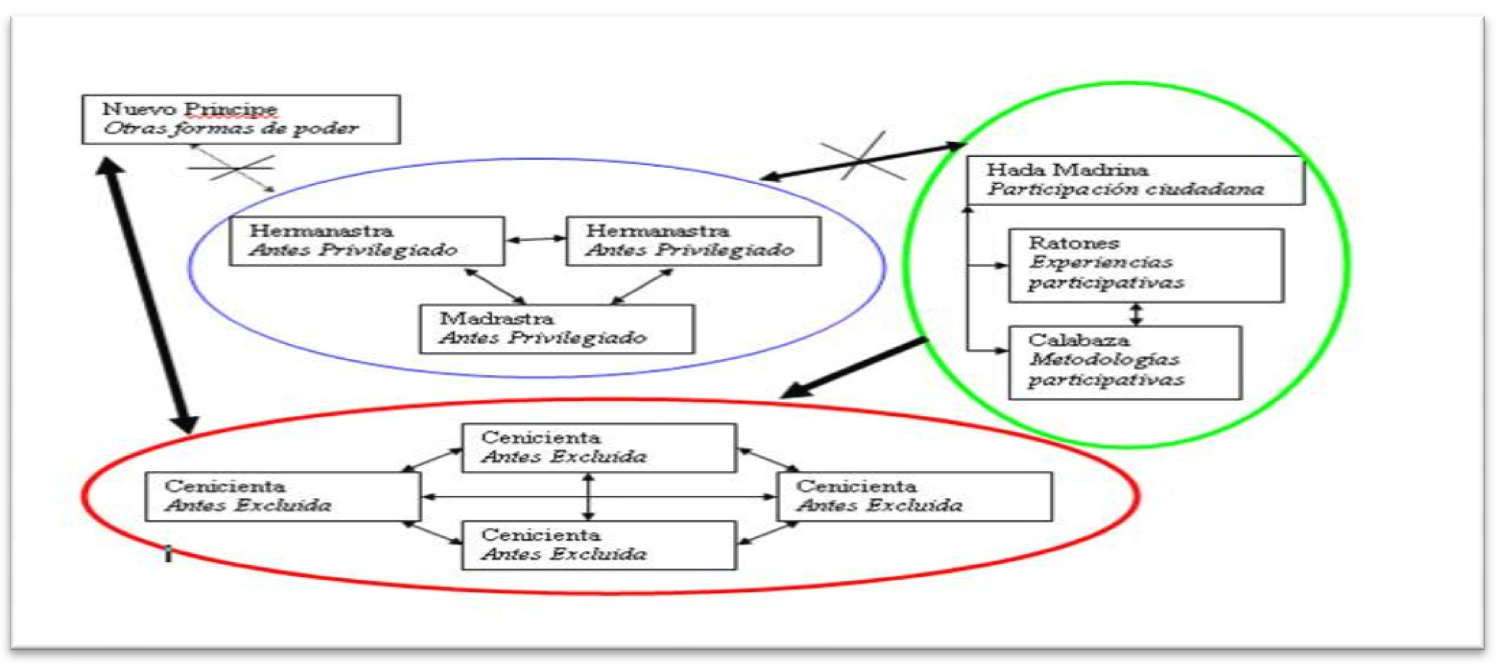

Fig. 1: Imagen utilizada en clase para ejemplificar el cuento de La Cenicienta

Situado el proceso de politización, se ejemplifica con la violencia contra las mujeres. Se reflexiona sobre la diferencia de sentido que subyace a los conceptos de violencia doméstica (que remite a lo privado y a salidas individuales) y violencia de género (de carácter estructural orientado a respuestas públicas y políticas). Se apunta que las relaciones de poder alcanzan su máxima expresión cuando se interpretan de forma privada haciéndose invisibles (Foucault, 2002) para las personas afectadas. El poder más eficaz, se concluye, es el que no se ve (Millet, 1970). El que se asume como "normal" (Allen, 1999). Sin embargo, Khaneman (2000) ha demostrado cómo los sesgos de control y de validez orientan nuestra percepción hacia una ilusión de inmunidad. Esto está presente en el alumnado de $1^{\circ}$, que en los test realizados considera que no se ve afectado por la desigualdad. A diferencia de lo que sucede en $4^{\circ}$ curso, donde las mujeres la identifican claramente tanto en los test como en los grupos de discusión. La clave son 4 años de experiencia de desigualdad. Por eso es necesario desvelarla al cuanto antes. Y la mejor forma es la práctica.

\section{Resultados: la desigualdad en la práctica}

La utilización de un cuento para presentar la asignatura es el resultado de una estrategia docente tendente a seducir al alumnado desde el primer momento. Se asume que la metodología a implementar es poco conocida y que su exigencia genera desconfianza. Por ello, el día de la presentación, junto a la del cuento, se combinan estrategias que desplieguen un efecto halo (Khaneman, 2000) que predisponga una actitud receptiva en el alumnado (García, 2002). Tras presentar los contenidos, se propone experimentar la metodología con una simulación de las estrategias que se utilizarán (juego de preguntas, Phillips 666, puzzle de debate) para mostrar un modelo en el que el protagonismo estará en su capacidad de trabajar en grupo, organizarse, repartir roles y gestionar tiempos para buscar soluciones grupales. La dinámica comienza con movimiento y se justifica como técnica de diagnóstico grupal: el alumnado se debe repartir en base a preguntas sobre su color de pelo, origen, tipo de estudios, lengua materna y, finalmente, género. Situados en dos filas, se escogen 3 personas de cada una para crear grupos mixtos de 6 personas, reclutándose a las excedentes (y las personas no binarias) como observadores a quienes se pide rellenar discretamente una ficha que mide la participación en términos de género. A ellos se les explica el sentido 
de la dinámica (visibilizar las desigualdades), convirtiéndoles en protagonistas de una dinámica de observación directa. Con la metodología Phillips 666 se les pide que a los grupos que identifique 6 palabras sobre política. Al no saber para qué es, algunos grupos negocian y otros hacen un sumatorio.

Después se pide que hagan una definición con ella. Quienes han negociado las palabras pueden hacer una buena definición, mientras que en el caso contrario suele carecer de sentido. Esta diferencia de comportamiento sirve para explicar que el trabajo cooperativo no es un sumatorio de trabajos individuales. Finalmente, se reparten a otros grupos y se pide que las valoren en grupo y expongan públicamente las críticas. Entre tanto, las personas del grupo de control miden los tiempos empleados en hablar por chicos y chicas en cada una de las tres dinámicas, quién propone, quién rechaza, quién corta, quién retoma y quién escribe. Las 3 experiencias realizadas entre 2018 y 2020 reflejan el mismo patrón: un mayor peso en la participación de las chicas (60\% del total) en la primera dinámica (de carácter privado y reproductivo, al tener que poner en acción habilidades de gestión de tiempo, de organización, de deliberación); de los chicos (60\%) en la segunda (de carácter pública y productiva, al concretarse en la redacción de una definición). Si en la primera de las dinámicas el peso de la metodología está en el expertise emocional; en la segunda lo está en el racional. Finalmente, en el debate público se da una aplastantemente mayoritaria de uso de la palabra por parte de los varones (90\% del total del tiempo). En las experiencias realizadas en 2018 y 2019, en el 100\% de las ocasiones las chicas redactaron la definición. Apoyados en el software Menti se pide que definan con una palabra el papel de la chicas en esta dinámica. En 2020 la más utilizada fue "secretaria".

Con esa estrategia, hemos logrado que la desigualdad tome cuerpo en el aula. Y que la sensación a la que habían llegado alumnas feministas que estaban en cuarto curso, se haga explícita desde el primer día del segundo cuatrimestre de $1^{\circ}$. En días posteriores, se retoma la discusión y se aprovecha para ejemplificar los acercamientos teóricos sobre el peso de las normas de género. Para ello, se les pide que respondan a una serie de test en los que se valoran competencias de trabajo en grupo, desigualdades en el aula, prejuicios y los resultados se devuelven identificando las variables en las que se hace evidente una valoración diferenciada por razón de género. Estas diferencias en la valoración de los liderazgos, la capacidad de empatizar, el sentido de la responsabilidad, el peso de lo individual o lo grupal, etc. son analizadas a partir de las normas de género. Los siguientes gráficos muestra los resultados obtenidos en 2020 respecto de: a) la valoración de las tareas de trabajo en grupo (Fig. 1); b) competencias del trabajo en grupo (Fig. 2); y c) la distribución de tiempos de la dinámica Phillips 666 (Fig. 3). 


\begin{tabular}{|c|c|c|c|c|c|c|c|c|c|c|c|c|}
\hline Se te pide que reflexiones el itinerario de trabajo del grupo al que & & & & & & Chicas & Chicos & & & & & \\
\hline $\begin{array}{l}\text { perteneces. Señala con una }(X) \text { la valoración que haces según el } \\
\text { esquema siguiente: } \\
1=\text { Nada } \\
2 \text { y } 3=\text { Escasamente } \\
4=\text { Algo } \\
56=\text { Mucho } \\
7=\text { Totalmente }\end{array}$ & Mini & \begin{tabular}{|l|l|} 
Coef \\
Variacion
\end{tabular} & \begin{tabular}{|l|} 
Desv \\
Estandar
\end{tabular} & Vatianza & Mediana & Media & Media & Mediana & \begin{tabular}{|l|l} 
Varianza \\
\end{tabular} & \begin{tabular}{|l|} 
Des \\
standar
\end{tabular} & $\begin{array}{l}\begin{array}{l}\text { Coef } \\
\text { variacion }\end{array} \\
\end{array}$ & Mini \\
\hline $\begin{array}{l}\text { Al comienzo, el grupo comprueba que todos y cada uno de los miembros } \\
\text { entiende del mismo modo el objetivo del trabajo. }\end{array}$ & 4 & 0,16 & 0,91 & 0,83 & 6 & 5,85 & 5,03 & 5 & 1,89 & 1,38 & 0,27 & 2 \\
\hline $\begin{array}{l}\text { Se determina entre todos el método y los procedimientos de realización } \\
\text { del trabajo. }\end{array}$ & 4 & 0,20 & 1,08 & 1,17 & 6 & 5,43 & 5,51 & 6 & 0,90 & 0,95 & 0,17 & 4 \\
\hline Definimos y formulamos entre todos el esquema del trabajo. & 5 & 0,13 & 0,85 & 0,71 & 7 & 6,29 & 5,37 & 5 & 0,82 & 0,90 & 0,17 & 4 \\
\hline $\begin{array}{l}\text { Se definen entre todos las funciones } y \text { tareas que se precisan para hacer } \\
\text { adecuadamente el trabajo. }\end{array}$ & 3 & 0,22 & 1,32 & 1,73 & 6 & 5,95 & 5,42 & 5,5 & 1,74 & 1,32 & 0,24 & 1 \\
\hline Distribuimos las funciones y tareas entre los miembros del grupo. & 4 & 0,16 & 1,03 & 1,06 & 7 & 6,55 & 5,86 & 6 & 1,05 & 1,03 & 0,18 & 4 \\
\hline Cada miembro del grupo realiza la tarea asignada en los plazos previstos & 2 & 0,21 & 1,29 & 1,67 & 7 & 6,1 & 5,86 & 6 & 1,41 & 1,19 & 0,20 & 3 \\
\hline $\begin{array}{l}\text { Ponemos en común las aportaciones individuales, para que todos y cada } \\
\text { uno tengan toda la información. }\end{array}$ & 4 & 0,14 & 0,87 & 0,76 & 7 & 6,43 & 5,66 & 6 & 1,19 & 1,09 & 0,19 & 3 \\
\hline $\begin{array}{l}\text { Las aportaciones individuales se debaten para elaborar un contenido } \\
\text { teórico o práctico común, suficientemente fundamentado y razonado. }\end{array}$ & 4 & 0,16 & 0,94 & 0,89 & 6 & 5,9 & 5,72 & 6 & 1,19 & 1,09 & 0,19 & 3 \\
\hline $\begin{array}{l}\text { Exponemos las distintas opiniones con argumentos, razones o datos } \\
\text { verificados. }\end{array}$ & 4 & 0,16 & 0,91 & 0,83 & 6 & 5,67 & 5,75 & 6 & 1,14 & 1,07 & 0,19 & 3 \\
\hline $\begin{array}{l}\text { Todos los participantes tienen la posibilidad de expresar y defender su } \\
\text { opinión. }\end{array}$ & 4 & 0,12 & 0,82 & 0,67 & 7 & 6,6 & 6,24 & 7 & 0,90 & 0,95 & 0,15 & 4 \\
\hline Todos participan prácticamente en los debates y discusiones. & 4 & 0,19 & 1,09 & 1,19 & 6 & 5,85 & 5,31 & 6 & 1,76 & 1,33 & 0,25 & 2 \\
\hline $\begin{array}{l}\text { Se comprueba si todos estamos de acuerdo con la reelaboración teórica, } \\
\text { o la resolución del problema realizados. }\end{array}$ & 4 & 0,16 & 0,90 & 0,81 & 6 & 5,71 & 5,13 & 5 & 0,82 & 0,90 & 0,18 & 4 \\
\hline $\begin{array}{l}\text { Intervenimos todos en la resolución del problema o en la elaboración de } \\
\text { las conclusiones de la cuestión. }\end{array}$ & 3 & 0,24 & 1,35 & 1,83 & 6 & 5,67 & 4,96 & 5 & 1,60 & 1,26 & 0,25 & 2 \\
\hline $\begin{array}{l}\text { Si no se está de acuerdo, se busca el modo de mejorar el texto o la } \\
\text { solución a fin de que todos den su conformidad. }\end{array}$ & 3 & 0,21 & 1,30 & 1,69 & 7 & 6,1 & 5,79 & 6 & 0,75 & 0,87 & 0,15 & 4 \\
\hline $\begin{array}{l}\text { Se verifica que todos sabrian responder adecuadamente del trabajo } \\
\text { realizado o resolver un problema parecido. }\end{array}$ & 3 & 0,21 & 1,21 & 1,46 & 6 & 5,81 & $\mathbf{5}$ & 5 & 1,31 & 1,15 & 0,23 & 2 \\
\hline $\begin{array}{l}\text { Evaluamos en el grupo los logros que vamos consiguiendo o el recorrido } \\
\text { realizado }\end{array}$ & 3 & 0,19 & 1,08 & 1,16 & 6 & 5,71 & 5,1 & 5 & 1,15 & 1,07 & 0,21 & 2 \\
\hline
\end{tabular}

Fig 2: Resultados en $1^{\circ}$ de Ciencia Política en el curso 2019/2020: Valoración de las tareas de trabajo en grupo

\begin{tabular}{|l|l|l|}
\hline Lo paso mal hablando en público (V/F) & OS & AS \\
\hline Tiendo a no responder (V/F) & 1,45 F & 1,31 F \\
\hline Liderar a otros/as (1-5) & 1,35 F & 1,05 F \\
\hline Participación activa (1-5) & 3,3 & 3,5 \\
\hline Toma de decisiones (1-5) & 4,06 & 3,90 \\
\hline Manejar conflictos (1-5) & 4,1 & 3,9 \\
\hline Pedir un favor $(1-5)$ & 3,55 & 3,71 \\
\hline
\end{tabular}

Fig 3: Resultados en $1^{\circ}$ de Ciencia Política en el curso 2019/2020: Valoración de las competencias de trabajo en grupo

\section{Conclusiones: avanzando en el aprendizaje basado en proyectos con perspctiva de género}

Considerada una "innovación disruptiva" en el ámbito educativo (Christensen et al., 2008: 214-215), la metodología del Abp presenta algunas fortalezas (Maida, 2011). Entre ellas, destaca que el alumnado adquiere la centralidad como sujeto de su aprendizaje (Lampert et al., 2013) al asumir un papel proactivo en la resolución de problemas (Abdullatif, 2019) mediante la promoción de la búsqueda autónoma de soluciones novedosas (Bilbao et al., 2018) a través de la colaboración (Russell, 2009; Al-Rahmi et al., 2015;). Aprovechando estas oportunidades, diversos estudios han planteado estas metodologías como instrumento para abordar los ODS en educación superior (Miñano y García, 2020; Unesco, 2017). Este aspecto es relevante, habida cuenta del creciente compromiso del ecosistema universitario en la incorporación de estos objetivos a la vida universitaria (https://www.crue.org/?s=ODS). Ahora bien, la incorporación de uno de los objetivos como es el de la igualdad de género, puede resultar lejano a los contenidos curriculares de ciertas disciplinas. Sin embargo, su inserción en las metodologías cooperativas permitiría integrarlo a las competenias tranversales de cualquier materia. Así. se considera que las estrategias de aprendizaje orientadas a la acción (Mezirow, 2000; Slavich y Zimbardo, 2012; Lotz-Sisitka,

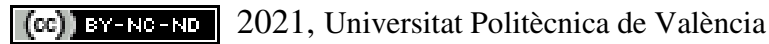

Congreso In-Red (2021) 
2015; Barthm 2015) invitan al alumnado a asumir un papel proactivo sobre cuestiones sociales, en tre ellas las de la igualdad de género.

En nuestro caso, nos centramos en detectar y gestionar las desigualdades aprovechando el proyecto propuesto para la evaluación final de la asignatura. Así, el diseño, que debe ser meticuloso (García, 2002; Markham, 2003), no solo contempla las actividades cooperativas y de autoaprendizaje, sino también los mecanismos de auto-organización que permiten consensuar normas de funcionamiento y evaluación, que son puestos al servicio de esta estrategia. Para lograr los objetivos, una clave de bóveda es el principio de interdependencia (Kagan, 1992) que obliga al grupo a velar por el avance en los resultados colectivos. Éste se puede alcanzar a través de diferentes herramientas, de entre ellas: 1. El convenio de grupo, según el cual se debe asumir colectivamente no sólo la obligación de trabajar sino el reparto de roles, la definición de los mecanismos de gestión de conflictos así como los sistemas de autoevaluación grupal. Este convenio puede ser firmado y acompañarse de una foto que visibilice unidad (ver Fig. 4) y colaboración a fin de que se entienda como un pacto de funcionamiento que defina compromisos individuales sometidos a evaluación por el resto de compañeros/as.

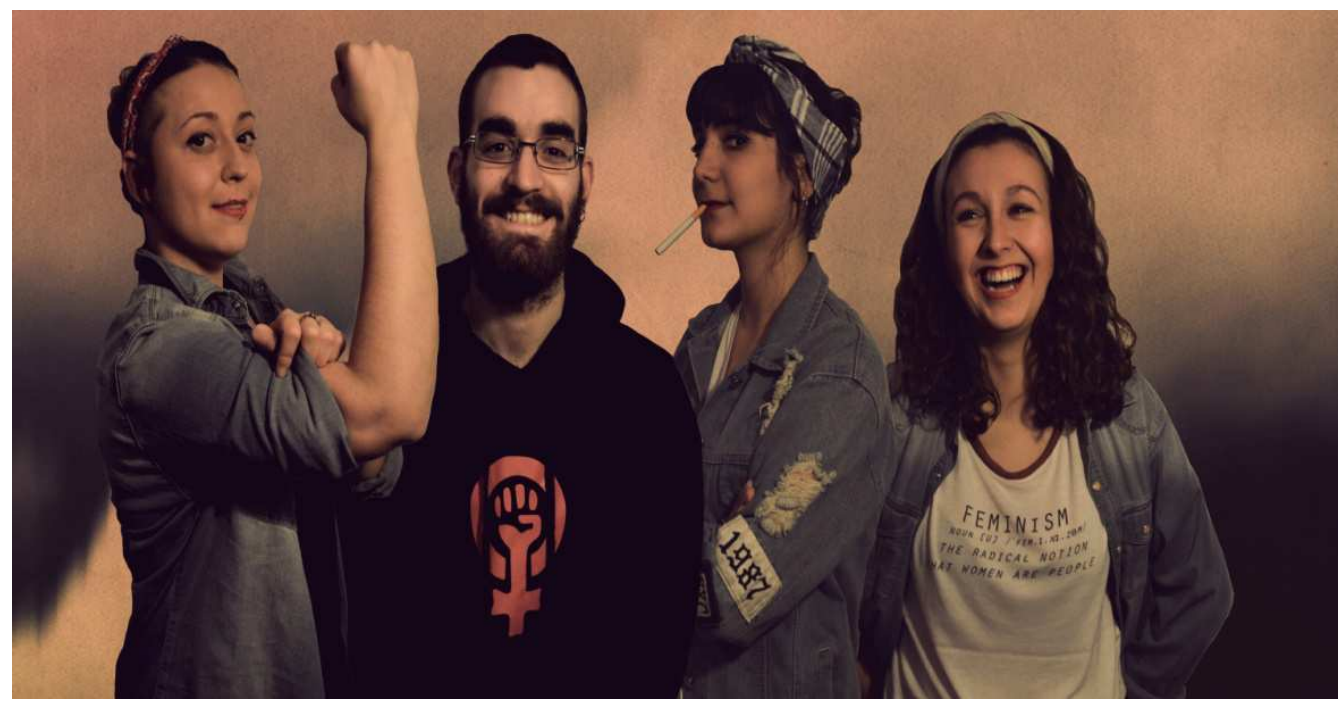

Fig 4: Fotografía de uno de los convenios de grupo ( $4^{\circ}$ curso, 2016/2017)

En nuestra propuesta, se plantea que cada grupo identifique los elementos diferenciales desvelados por los cuestionarios y se les invita comprometerse con la perspectiva de género en el desarrollo del trabajo. 2. El cuaderno de viaje, que permite narrar los avances y obstáculos tanto en los contenidos como en el proceso haciendo posible centrar la atención en la desigualdad durante las reflexiones semanales. 3. Una rúbrica de tiempos para el trabajo en grupo (elaborada por las alumnas de cuarto curso) que se les invita a utilizar como mecanismo de control de las desigualdades que pudieran reproducirse en cada grupo. Estos elementos sirven de auto-evaluación para los componentes del grupo.

Como hemos comprobado y pretendemos profundizar, al situar al alumnado en el centro del proceso, el Abp emerge como una metodología idónea para abordar las desigualdades. El uso de estrategias reflexivas autónomas asentadas en la observación, la sistematización y la reflexión unidas a mecanismos de gestión, se añade a la labor de acompañamiento del profesorado. Este modelo aporta una mirada cualitativa y resolutiva a investigaciones cuantitativas que identifican patrones desiguales en relación al género en el aula (Bolívar-Cruz et al., 2018). El peso de las normas de género se evidencia a través de 
dinámicas que revelan una distribución desigual y estereotípica del trabajo grupal y los roles asociados al mismo. La comprensión de las raíces públicas de problemas previamente vistos como privados visibiliza que "el sexo importa" (Pateman, 1990) cuando estas desigualdades son identificadas a través de los cuestionarios proporcionados y las dinámicas desarrolladas. Los resultados de esta experiencia didáctica subrayan la necesidad de politizar la mirada privada a la desigualdad desde la experiencia, con el fin de preparar al alumnado para comprender(se en) las estructuras, los procesos y los resultados en la esfera de lo político.

Considerando los límites experimentales de la experiencia presentada, la continuidad de esta propuesta requerirá:

- Validar los cuestionarios de diagnóstico como instrumentos para el análisis de las desigualdades de género en el aula en grupos más amplios para recabar las suficientes evidencias en relación a la calidad de su diseño.

- Incorporar la perspectiva interseccional (Hill Collins y Bilge, 2019) para captar lógicas de desigualdad asociadas a variables que influyen en el Abp como la clase o la raza (Hirshfiel y Koretsky, 2017) al entender que "cuanto más se investiga sobre diversidad, mas prácticas inclusivas se pueden poner en marcha" (Llorent et al., 2020).

- Ejecutar una observación más sistematizada de las dinámicas implementadas en cada grupo.

- Capacitar a los y las docentes en las cualidades y condiciones que requiere la implementación de este modelo de aprendizaje.

Consideramos, en definitiva, que esa relación dialéctica que vincula los principios de la estrategia metodológica del Abp con los mecanismos que brinda la perspectiva de género abre una fecunda posibilidad para plantear una propuesta de abordaje pedagógico que garantice visibilización y autogestión de las relaciones desigualitarias por parte del alumnado en el marco de los trabajos grupales. Y esta experiencia puede ayudar a avanzar en otras disciplinas.

\section{Referencias}

ABDULLATIF, M. (2019). "The Effectiveness of the Project-Based Learning (PBL) Approach as a Way to Engage Students in Learning", en Sage Open, July-September 2020, p. 1-15. https://doi.org/10.1177/2158244020938702

AHEDO, I. (2016). "Piezas para comenzar a andar. Política, cenicientas y reinos trasnparentes. Tema 0. Fundamentos de Análisis Político", en Open Course Ware. UPV/EHU. https://ocw.ehu.eus/pluginfile.php/49852/mod_resource/content/5/Tema\%200.pdf

AHEDO, I., MARTINEZ-PALACIOS, J., MARTIZ-PORTUGAL, T (2017). Evaluación cualitativa de la Ley 4/2005 para la igualdad de mujeres y hombres en la CAE. 2015. Vitoria-Gasteiz: Gobierno Vasco Emakunde.

https://www.emakunde.euskadi.eus/contenidos/informacion/politicas_evaluaciones/es_def/adjuntos/2015. evaluacion_cualitativa.pdf

ALLEN, A. (1999). The Power of Feminist Theory. Boulder: Westview Press.

AL-RAHMI, W.M., OTHMAN, M. S. y YUSUF, L.M. (2015). "Exploring the factors that affect student satisfaction through using e-learning in Malaysian higher education institutions", en Mediterranean Journal of Social Sciences, 6 (4), p. 299-310. https://doi.org/10.5901/mjss.2015.v6n4s1p299

(c)) EY-NC-ND 2021, Universitat Politècnica de València

Congreso In-Red (2021) 
BARTH, M. (2015). Implementing Sustainability in Higher Education. London: Routledge. https://doi.org/10.4324/9780203488355

BENGOECHEA, M. (2015). "Las buenas alumnas ante los TFG: atrapadas entre la cultura comunicativa femenina y el androcentrismo", en Libro de Actas de la III Xornada de Innovación en Xénero, Docencia e Investigación. Vigo: Universidade de Vigo, Unidade de Igualdade, p. 9-35.

BENITO, E. y VERGE, T. (2020). "Gendering higher education quality assurance: a matter of (e)quality" en Quality in Higher Education. Epub. June, 10. https://doi.org/10.1080/13538322.2020.1769268

BILBAO, J. et al. (2018)." Selecting assessments for problem based learning”, en International Journal of Education and Learning Systems, 3, p. 129-133.

BOLIVAR-CRUZ, A., VERANO-TACORONTE, D. y GALVAN-SANCHEZ, I. (2018). "Do selfefficacy, incentives and confidence in public speaking influence how students self-asses? / Influyen la autoeficacia, los incentivos y la confianza para hablar en público en cómo se autoevalúan los estudiantes", en Cultura y Educación, 30 (3), p. 528-555. https://doi.org/10.1080/11356405.2018.1488420

BOURDIEU, P. (1988). La distinción: Criterios y bases sociales del gusto. Madrid: Taurus.

BOURDIEU, P. (2000). La dominación masculina. Barcelona: Anagrama.

BUTLER, J. (1997). Mecanismos psíquicos del poder: Teorías sobre la sujeción. Madrid: Cátedra.

CAMINAL, M. (2006). Manual de ciencia política. Madrid: Tecnos

CARRASCO, C. (1992). "El trabajo de las mujeres: producción y reproducción (algunas notas para su reconceptualización)", en Cuadernos de economía: Spanish Journal of Economics and Finance, 20 (5758), p. 95-109. http://hdl.handle.net/10486/5639

CASSESE, E.C. y BOS, A.L. (2013). “A hidden curriculum? Examining the gender content in introductory-level political science textbooks", en Politics and Gender, 9 (2), p. 214-223. https://doi.org/10.1017/S1743923X13000068

CHRISTENSEN, C.M., HORN, M.B. y JOHNOSON, C.W. (2008). Disrupting Class: how disruptive innovation will change the way the world learns. New York: McGraw-Hill.

COHEN, J. y ARATO, A. (1992). Civil society and political theory. Cambridge: MIT Press.

CONSEJO DE EUROPA (2007). Recomendación CM/Rec del Comité de Ministros a los Estados miembros sobre las normas y los mecanismos de igualdad entre mujeres y hombres. https://www.inmujer.gob.es/publicacioneselectronicas/documentacion/Documentos/DE0019.pdf

De LAURETIS, T. (1989). Technologies of Gender: Essays on Theory, Film and Fiction. Londres: Macmillan Press.

DEL CASTILLO, O. y CORRAL, J.A. (2014). "El profesorado frente a la discriminación de género: uso de la retroalimentación", en Cultura y Educación, 23 (4), p. 487-498. https://doi.org/10.1174/113564011798392415 
De LOTTELL, P.J., MILlAM, L.A., y REINHARDT, M.M. (2010). "The Use Of Deep Learning Strategies In Online Business Courses To Impact Student Retention", en American Journal of Business Education (AJBE), 3 (12), p. 49-56. https://doi.org/10.19030/ajbe.v3i12.964

DOYLE, T. (2008). Helping students learn in a learner-centered environment a guide to facilitating learning in higher education (1st ed.). Sterling.: Stylus Pub.

ESTEBAN, M.L. (2004). Antropología del cuerpo: Género, itinerarios corporales, identidad y cambio. Barcelona: Edicions Bellaterra.

FEDERICI, S. (2010). Caliban y la bruja. Mujeres, cuerpo y acumulación originaria. Madrid: Traficantes de sueños. https://www.traficantes.net/sites/default/files/pdfs/Caliban\%20y\%20la\%20bruja$\underline{\text { TdS.pdf }}$

FOUCAULT, M. (2002). Vigilar y castigar. Buenos Aires: Siglo XXI.

FREIXAS, A. y FUENTES-GUERRA, M. (1997). "Haciendo visible el género en el aula: clima de clase y acción del profesorado", en Cultura y Educación, 9 (4), p. 13-25. https://doi.org/10.1174/113564097760624720

GARCIA, J.N. (2002). "El aprendizaje basado en problemas: una ilustración de un modelo de aplicaciones en psicopedagogía", en Cultura y Educación, 14 (1), p. 65-79. https://doi.org/10.1174/113564002317348129

GARCÍA-PEREZ ET AL. (2011). "El patriarcado no es transparente: competencias del profesorado para reconocer la desigualdad", en Cultura y Educación, 23 (3), p. 385-397. https://doi.org/10.1174/113564011797330298

GILLESPIE, D., ROSAMOND, S. y THOMAS, E. (2006b). "Grouped out? Default strategies for participating in multiple small groups", en Journal of General Education, 55 (2), p. 81-102. https://doi.org/10.1353/jge.2006.0022

GILLESPIE, D., ROOS, J. y SLAUGHTER, C. (2006a). "Ambivalence about leadership in small groups", en Journal of Excellence in College Teaching, 17 (3), p. 33-49.

HERNANDO, A. (2018). La fantasía de la individualidad. Sobre la construcción socio-histórica del sujeto moderno. Madrid: Traficantes de sueños. https://www.traficantes.net/sites/default/files/pdfs/map50_la\%20fantasia_web.pdf

HILL COLLINS, P. y BILGE, S. (2019). Interseccionalidad. Madrid: Morata.

HILLYARD, C., GILLESPIE, D. y LITTING, P. (2010). "University students' attitudes about learning in small groups after frequent participation", en Active Learning in Higher Education, 11(1), p. 9-20. https://doi.org/10.1177/1469787409355867

HIRSHFIELD, L. y KORETSKY, M.D. (2018). "Gender and Participation in an Engineering ProblemBased Learning Environment", en Interdisciplinary Journal of Problem-Based Learning, 12 (1). https://doi.org/10.7771/1541-5015.1651 
ISAKSEN, L.W., DEVI, S.U. y HOCHSCILD, A.R. (2008). "Global Care Crisis: A Problem of Capital, Care Chain, or Commons?”, en American Behavioral Scientist, 52 (3), p. 405-425. https://doi.org/10.1177/0002764208323513

KAGAN, S. (1992). Cooperative learning. San Juan Capistrano: Resources for teachers, Inc.

LAMAS, M. (2002). Cuerpo: Diferencia sexual y género. Madrid: Aguilar.

España. Ley Orgánica 4/2007, de 12 de abril, por la que se modifica la Ley Orgánica 6/2001, de 21 de diciembre, de Universidades. https://www.boe.es/eli/es/lo/2007/04/12/4

LIDEN, R.C., NAGAO, D.H. y PARSO C.K. (1985). "Student and faculty attitudes concerning the use of group projects", en Organizational Behavior Teaching Review, 10 (4), p. 32-8. https://doi.org/10.1177/105256298601000403

LIZZIO, A. y WILSON, K. (2005). "Self-managed learning groups in higher education: Students' perceptions of process and outcomes", en British Journal of Educational Psychology, 75 (3), p. 373-90. https://doi.org/10.1348/000709905X25355

LLORENT, V.J., ZYCH, I. y VARO-MILLAN, J.C. (2020). "University academic personnet's visión of inclusive education in Spanish universities / Visión del profesorado sobre la educación inclusiva en la universidad de España", en Cultura y Educación, 31 (1), p. 147-181. https://doi.org/10.1080/11356405.2019.1705593

LOIS, M. y ALONSO, A. (2014.). Ciencia política con perspectiva de género. Barcelona: Akal.

LOTZ-SISITKA, H. et al. (2015). "Transformative, transgressive social learning: rethinking higher education pedagogy in times of systemic global dysfunction", en Current Opinion in Environmental Sustainability, 16, p. 73-80. https://doi.org/10.1016/j.cosust.2015.07.018

MAIDA, C.A. (2011). "Project-Based Learning: A Critical Pedagogy for the Twenty-First Century", en Policy Futures in Education, 9 (6), p. 759-768. https://doi.org/10.2304/pfie.2011.9.6.759

MARKHAM, T. (2003). Project-based Learning Handbook: a guide to standards-focused project-based learning for middle and high school teachers, 2nd ed. Novato: Buck Institute for Education.

MARTINEZ-PALACIOS, J. (2018b). No te pongas nerviosa. Pamplona: Pamiela.

MARTINEZ-PALACIOS, J. Coord. (2018a). Innovaciones democráticas feministas. Madrid: Dykinson.

MARTINEZ-PALACIOS, J. (2017a). "Inclusive democratization: normative proposals and political practices", en Local Government Studies, $43 \quad$ (4) p. 577 597. http://dx.doi.org/10.1080/03003930.2017.1303485

MARTINEZ-PALACIOS, J. (2017b). "Contra-públicos feministas e innovaciones democráticas. Estrategias para una profundización democrática inclusiva”, en Revista de Estudios Políticos, 178, p. 105136. https://doi.org/10.18042/cepc/rep.178.04

MEZIROW, J. (2009). Transformative Learning in Practice: Insights from Community: Insights from Community, Workplace, and Higher Education. Nueva York: Jossey-Bass Inc. 
MICARI, M. y PAZOS, P. (2014). "Worrying about what others think: A social-comparison concern intervention in small learning groups", en Active Learning in Higher Education, 15 (3), p. 249-262. https://doi.org/10.1177/1469787414544874

MILLET, K. (1970). Política sexual. Madrid: Cátedra.

MIÑANO, R. y GARCÍA, M. (2020). Implementando la agenda 2030 en la Universidad. Casos inspiradores. Madrid: REDS.

MOSS-RACUSIN, C.A. et al. (2012). "Science faculty's subtle gender biases favor male students", en PNAS October 9, 109 (41), p. 16474-16479. https://doi.org/10.1073/pnas.1211286109

ORMAZABAL, A. y GOROSTIDI, I. (2017). "Orientaciones para trabajar las asimetrías de poder en la participación social y política”, en MARTINEZ-PALACIOS, J. (Coord.), Participar desde los feminismos. Ausencias, expulsiones y resistencias (p. 235-286). Barcelona: Icaria.

PATEMAN, C. (1990). 'Does Sex Matter to Democracy?'- A Comment, en Scandinavian Political Studies, 13 (1), p. 57-63. https://doi.org/10.1111/j.1467-9477.1988.tb00373.x

PRADOS, M. y CUBERO, M. (2013). "Reflexionando acerca del cómo estudiar el proceso de enseñanzaaprendizaje en el contexto natural del aula universitaria", en Cultura y Educación, 25 (3), p. 273-284. https://doi.org/10.1174/113564013807749696

PUJOLAS, P. (2009). "La calidad en los equipos de aprendizaje cooperativo. Algunas consideraciones para el cálculo del grado de cooperatividad”, en Revista de Educación, 349. Mayo-agosto 2009, p. 225239.

RD 1393/2007, de 29 de octubre por el que se establece la ordenación de las enseñanzas universitarias oficiales. Establece el sistema de enseñanzas universitarias oficiales de España, y el procedimiento de evaluación para su verificación, seguimiento y acreditación. https://www.boe.es/buscar/act.php?id=BOE$\underline{\mathrm{A}-2007-18770}$

ROBINSON, L., HARRIS, A. y BURTON, R. (2015). "Saving face: Managing rapport in a problem based learning group", en Active Learning in Higher Education, 16 (1), p. 11-24. https://doi.org/10.1177/1469787415573355

RUSSELL, D. (2009). "Group collaboration in an online problem based university course", en TAN, O.S. (Ed.), Problem-based learning and creativity (pp. 173-192). Singapur: Cengage Learning Asia.

SEGATO, R.L. (2016). La guerra contra las mujeres. Madrid: Traficantes de Sueños.

SLAVICH, G.M. y ZIMBARDO, P.G. (2012). "Transformational Teaching: Theoretical Underpinnings, Basic Principles, and Core Methods", en Educational Psychology Review, 24 (4), p 569-608. https://doi.org/10.1007/s10648-012-9199-6

SWANSON, E. et al. (2019). "The effect of team-based learning on content knowledge: A metaanalysis", en Active Learning in Higher Education, 20 (1), p. 39-50. https://doi.org/10.1177/1469787417731201

UNESCO (2017). Educación para los objetivos de desarrollo sostenible. Objetivos de aprendizaje. París.

(cc) EY-NC-ND 2021, Universitat Politècnica de València

Congreso In-Red (2021) 
VALLES, J. M. (2006). Ciencia política, una introducción. Ariel: Barcelona.

VARELA, N. (2019). Feminismo 4.0. La cuarta ola. Barcelona: Ediciones B.

VERGE, T., FERRER-FONS, M. y GONZALEZ, M. (2018). "Resistances to Mainstreaming Gender into the Higher Education Curriculum”, en European Journal of Women's Studies, 25 (1), p. 86-101. https://doi.org/10.1177/1350506816688237

YOUNG, I.M. (2002). Inclusion and Democracy. Oxford: Oxford University Press.

ZUGAZA, U. et al. (2020). "La inclusión de la perspectiva de género en la docencia de la Universidad del País Vasco: diagnóstico y propuestas”, en Revista de Estudios Empresariales. Segunda época, 2 (2019), p. 25-48. https://dx.doi.org/10.17561/ree.v2019n2.2 